\title{
SPH simulations of grain growth in protoplanetary disks
}

\author{
G. Laibe ${ }^{1}$, J.-F. Gonzalez ${ }^{1}$, L. Fouchet ${ }^{2}$, and S. T. Maddison ${ }^{3}$ \\ 1 Université de Lyon, Lyon, 69003; Université Lyon 1, Villeurbanne, 69622; CNRS, UMR 5574, Centre de Recherche Astrophysique \\ de Lyon; École Normale Supérieure de Lyon, 46 allée d'Italie, 69364 Lyon Cedex 07, France \\ e-mail: [Guillaume.Laibe; Jean-Francois.Gonzalez]@ens-lyon.fr \\ 2 Institute of Astronomy, ETH Zürich, Schafmattstrasse 16, HPT D19, 8093 Zürich, Switzerland \\ e-mail: fouchet@phys.ethz.ch \\ ${ }^{3}$ Centre for Astrophysics and Supercomputing, Swinburne Institute of Technology, PO Box 218, Hawthorn, VIC 3122, Australia \\ e-mail: smaddison@swin.edu.au
}

Received 6 February 2008 / Accepted 5 June 2008

\section{ABSTRACT}

\begin{abstract}
Aims. In order to understand the first stages of planet formation, when tiny grains aggregate to form planetesimals, one needs to simultaneously model grain growth, vertical settling and radial migration of dust in protoplanetary disks. In this study, we implement an analytical prescription for grain growth into a 3D two-phase hydrodynamics code to understand its effects on the dust distribution in disks.

Methods. Following the analytic derivation of Stepinski \& Valageas (1997, A\&A, 319, 1007), which assumes that grains stick perfectly upon collision, we implement a convenient and fast method of following grain growth in our 3D, two-phase (gas+dust) SPH code. We then follow the evolution of the size and spatial distribution of a dust population in a classical T Tauri star disk. Results. We find that the grains go through various stages of growth due to the complex interplay between gas drag, dust dynamics, and growth. Grains initially grow rapidly as they settle to the mid-plane, then experience a fast radial migration with little growth through the bulk of the disk, and finally pile-up in the inner disk where they grow more efficiently. This results in a bimodal distribution of grain sizes. Using this simple prescription of grain growth, we find that grains reach decimetric sizes in $10^{5}$ years in the inner disk and survive the fast migration phase.
\end{abstract}

Key words. planetary systems: protoplanetary disks - hydrodynamics - methods: numerical

\section{Introduction}

The first steps of planet formation are governed by the build-up of planetesimals due to the dust coagulation in protoplanetary disks (Dominik et al. 2007). Observational evidence for grain growth in disks is now common (Apai et al. 2004; Rodmann et al. 2006; Muzerolle et al. 2006; Lommen et al. 2007; Graham et al. 2007). Grains must grow from sub- $\mu \mathrm{m}$ sizes to planetesimal scale (kilometer size) objects in a fraction of the lifetime of the disk, which is estimated to be a few $10^{7}$ years (Haisch et al. 2001; Carpenter et al. 2005). The timescales of grain growth, however, are unclear: some young disks show signatures of grain growth while old disks can show signatures of unprocessed grains and coeval disks can show a range of grain sizes and dust processing (Kessler-Silacci et al. 2006).

Grains can grow via collisions and depending on their relative velocity and on their chemical and physical properties (Chokshi et al. 1993; Blum 2006), colliding grains can rebound, shatter or stick. Grains will settle vertically and migrate radially at different rates according to their size (Weidenschilling 1977; Garaud et al. 2004; Barrière-Fouchet et al. 2005), leading to local density enhancements in the disk. Since grain growth is dependent on density, changes in the dust distribution will affect growth rates, which in turn will affect the dynamics of the dust (Weidenschilling 1980; Haghighipour 2005). Therefore, growth, settling and migration need to be simulated together.

Various models have been developed to describe the grain growth process. One approach is to use the time-dependent
Smoluchowski coagulation equation (Weidenschilling 1980, 1997; Suttner \& Yorke 2001; Dullemond \& Dominik 2005; Tanaka et al. 2005; Nomura \& Nakagawa 2006; Ciesla 2007) which describes the number density evolution of particles of a given mass range. The numerical solution of the Smoluchowski equation is challenging. Another approach is to use an analytic expression for the grain growth rate as a function of local disk conditions (Stepinski \& Valageas 1997; Haghighipour 2005). In this study, we use this second approach and implement the analytical prescription of Stepinski \& Valageas (1997, hereafter SV97) in our 3D, two-phase (gas+dust) hydrodynamics code and follow the evolution of the grain size distribution in a protoplanetary disk. We validate our method on an axisymmetric disk here, before applying it to non-axisymmetric complex problems in future work.

\section{An analytical grain growth model}

SV97 modeled the radial evolution of solid particles made of water ice in geometrically thin, turbulent, vertically isothermal protoplanetary disks. They described the gas and solid particle components as two separate phases coupled by aerodynamic forces (in the Epstein regime), and assumed that the evolution of the gas is unaffected by that of the solids. The particles can grow by coagulation (they stick perfectly upon collision and therefore never shatter into smaller grains), evaporate or condense from vapor. Their size distribution at any radius and time is supposed to be 
narrowly peaked around a local mean value $s(r, t)$. Self-gravity is neglected.

They obtained an analytic expression for the evolution of the particle size $s$ given by

$\frac{\mathrm{d} s}{\mathrm{~d} t}=\sqrt{2^{3 / 2} \operatorname{Ro} \alpha} \frac{\hat{\rho}_{\mathrm{d}}}{\rho_{\mathrm{d}}} C_{\mathrm{s}} \frac{\sqrt{\mathrm{Sc}-1}}{\mathrm{Sc}}$,

where Ro is the Rossby number for turbulent motions, $\alpha$ the Shakura \& Sunyaev (1973) viscosity parameter, $\hat{\rho}_{\mathrm{d}}$ the density of matter concentrated into solid particles, $\rho_{\mathrm{d}}$ the bulk density of the grains, $C_{\mathrm{s}}$ the local gas sound speed, and Sc the Schmidt number of the flow which estimates the effect of gas turbulence on the grains. They defined Sc as

$\mathrm{Sc}=\left(1+\Omega_{\mathrm{K}} t_{\mathrm{s}}\right) \sqrt{1+\frac{\overline{\boldsymbol{v}}^{2}}{V_{\mathrm{t}}^{2}}}$,

where $\Omega_{\mathrm{K}}$ is the local keplerian velocity, $t_{\mathrm{s}}$ the dust stopping time, $\overline{\boldsymbol{v}}$ the mean relative velocity between gas and dust, and $V_{\mathrm{t}}$ a turbulent velocity. Note that Youdin \& Lithwick (2007) recently suggested that one should use $\mathrm{Sc} \simeq 1+\left(\Omega_{\mathrm{K}} t_{\mathrm{S}}\right)^{2}$ instead. However it is not clear how this approximate value follows from their more complex exact expression $\left(1+\left(\Omega_{\mathrm{K}} t_{\mathrm{S}}\right)^{2}\right)^{2} /\left(1+4\left(\Omega_{\mathrm{K}} t_{\mathrm{S}}\right)^{2}\right)$. More importantly, the latter equation applies to diffusion of particles rather than to collision rates, for which Eq. (2) is appropriate. In this paper, we keep the formulation used by SV97 for the sake of self-consistency.

The growth rate $\frac{\mathrm{d} s}{\mathrm{~d} t}$ depends on $s$ via the stopping time

$t_{\mathrm{s}}=\frac{\rho_{\mathrm{d}} S}{\rho_{\mathrm{g}} C_{\mathrm{s}}}$,

where $\rho_{\mathrm{g}}$ is the gas density.

SV97 were mostly interested in the radial distribution of solid particles and their sizes. However, in order to interpret observations of disks showing evidence of grain settling and growth, as well as to provide initial conditions for planet formation models, which are normally axisymmetric situations, one needs to know both the radial and vertical size and density distributions of grains. The complex interplay between the drag force, which causes solids to migrate radially and settle vertically, and the growth process makes a full numerical treatment of this problem necessary.

\section{Grain growth in a 3D SPH code}

We have developed a 3D, two-phase (gas+dust) Smoothed Particles Hydrodynamics (SPH) code to model vertically isothermal, non self-gravitating protoplanetary disks. The two inter-penetrating phases representing gas and dust interact via aerodynamic drag. Barrière-Fouchet et al. (2005, hereafter $\mathrm{BF} 05$ ) describe the code and its limitations, and present the spatial distribution of dust grains ranging from $1 \mu \mathrm{m}$ to $10 \mathrm{~m}$ in size resulting from radial migration and vertical settling. In this work, our aim is to implement the grain growth algorithm of SV97 into our code and see how this simple prescription of grain growth affects the dust dynamics by comparing with the results of BF05. While this could adequately be tested in 2D, our ultimate goal is to study the observational signatures of grain growth in protoplanetary disks and apply the code to various non-axisymmetric problems, like disks with embedded planets. Thus we will be able to extend our previous work on the formation of planetary gaps in the dust layers of protoplanetary disks
(Maddison et al. 2007; Fouchet et al. 2007) to include grain growth, and study the stratification of growing dust grains in disks (see, e.g., Pinte et al. 2007).

SPH is very well suited to the SV97 implementation of grain growth. As we do in our code, SV97 describe gas and dust as two fluids, their disk configuration and thermodynamics are very similar to ours, and our SPH viscosity can be related to a Shakura-Sunyaev $\alpha$ viscosity (Fouchet et al. 2007). Our test simulations show that the gas disk is little affected by the evolution of the dust, as assumed by SV97. Therefore, it is straightforward to implement the SV97 prescription of grain growth, given by Eq. (1), in our code.

Following the work of SV97, all the dust particles must have the same initial size, $s_{0}$. The size $s$ is then evolved using Eq. (1) evaluated at the location of each SPH particle. We assume that the size $s$ assigned to each SPH particle represents the typical size of physical dust grains at its location in the disk, at a given time. Again, this is very similar to the assumption of SV97 for their local mean value $s(r, t)$. Contrary to their work, we do not take evaporation into account: for our disk conditions (see Sect. 4.1), SV97 show that the evaporation radius is located between 1 and $2 \mathrm{AU}$ from the star and that after a million years only a small fraction of the total solid material is evaporated due to migration. Similar to SV97, our implementation of grain growth does not include the fragmentation of grains, even though it is likely to play an important role (see Sect. 6).

The mass of each SPH particle is kept constant to ensure kinetic energy and momentum conservation. As $s$ can only increase, this implies that, over time, SPH dust particles represent fewer but larger physical dust grains. However, even for km-sized planetesimals, each SPH particle still represents a very large number of physical particles, maintaining the validity of the numerical scheme.

\section{Simulations}

\subsection{Setup}

We study grain growth in the typical $\mathrm{T}$ Tauri disk modeled in BF05 with $M_{\text {disk }}=0.02 M_{\odot}$, composed of $99 \%$ gas and $1 \%$ dust by mass and orbiting a $1 M_{\odot}$ star. The dust grains have an intrinsic density $\rho_{\mathrm{d}}=1 \mathrm{~g} \mathrm{~cm}^{-3}$. We choose an initial state for a gas disk near equilibrium conditions. We take the parameters of the Minimum Mass Solar Nebula for the radial dependence of the temperature $\left(T \propto r^{-3 / 4}\right)$ and initial surface density $(\Sigma \propto$ $\left.r^{-3 / 2}\right)$. The disk is locally isothermal, i.e. the temperature follows a radial power law but is vertically constant. The sound speed then varies as $C_{\mathrm{s}} \propto r^{-3 / 8}$ and, given that $H=C_{\mathrm{s}} / \Omega_{\mathrm{K}}, H / r$ varies as $r^{1 / 8}$. The disk is slightly flared with $H / r=0.05$ at $100 \mathrm{AU}$.

The smoothing length is computed by $h_{i} \propto\left(m_{i} / \rho_{i}\right)^{1 / 3}$, where $m_{i}$ and $\rho_{i}$ are the mass and density of an SPH particle (see BF05). The code will not be able to resolve a dust disk for which the semi-thickness is less than the smoothing length. However, turbulence in real disks stirs the solid particles and prevents the formation of such a thin dust layer. Indeed, the dust disk thickness reaches a steady state when the settling and turbulent mixing are in equilibrium (see, e.g., Dubrulle et al. 1995; Dullemond \& Dominik 2004; Schräpler \& Henning 2004). Our code does not yet include turbulent mixing and therefore cannot reproduce this steady state. The SPH artificial viscosity terms are given by $\alpha_{\mathrm{SPH}}=0.1$ and $\beta_{\mathrm{SPH}}=0.0$, which ensures the corresponding Shakura \& Sunyaev (1973) viscosity parameter $\alpha \sim 0.01$ (as indicated by observations of protoplanetary disks - see Hartmann et al. 1998; King et al. 2007). 


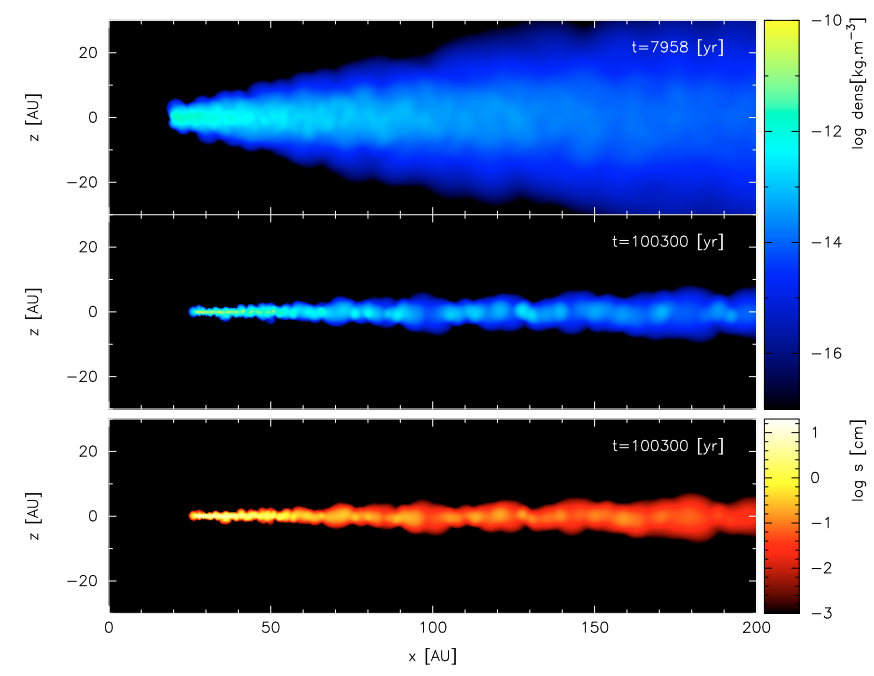

Fig. 1. Initial (top) and final (middle) dust density and final grain size distribution (bottom) in a meridian plane cut of the disk for $s_{0}=10 \mu \mathrm{m}$.

We start with 200000 gas particles that are distributed radially so as to retrieve the expected power law for surface density and randomly in the vertical direction because hydrostatic equilibrium is rapidly reached. The initial velocity of the gas particles is Keplerian. Starting from this initial distribution, we allow the gas disk to relax for almost 8000 years, which allows the pressure and artificial viscosity to smooth out the velocity field. Once the gas disk has relaxed, we then add an equal number of dust particles on top of the gas particles with the same velocity and allow the system to evolve.

The disk extends initially from 20 to 300 AU. During the evolution, particles are removed from the simulation if they migrate inside of $20 \mathrm{AU}$ and are assumed to be accreted by the star. The outer boundary is free and particles are only removed if they go beyond $400 \mathrm{AU}$. The viscous evolution of the disk is so slow that the gas surface density profile stays almost unchanged although the disk expands radially up to $400 \mathrm{AU}$.

We ran a series of simulations with $400000 \mathrm{SPH}$ particles and initial grain sizes $s_{0}$ ranging from $1 \mu \mathrm{m}$ to $1 \mathrm{~mm}$, in which the system is evolved for a total of $10^{5} \mathrm{yr}$. The results presented in the next sections are time snapshots of the ongoing disk evolution.

\subsection{Results}

Figure 1 shows the density of the solid phase at dust injection (top) and at the end of the simulation (middle), along with the resulting size distribution (bottom) in a meridian plane cut of the disk for $s_{0}=10 \mu \mathrm{m}$. The vertical profiles of the gas and dust densities at the end of the simulation are shown in more detail in Fig. 2. The very efficient settling produces a thin dust disk, whereas the gas disk does not evolve on this timescale.

Grain growth occurs very quickly: a few hundred years after dust injection, the innermost grains have almost reached $\mathrm{mm}$ sizes (Fig. 3a), and a few thousand years later (Fig. 3b), grain growth is visible over the entire disk, with sizes of a few $\mathrm{cm}$ in the inner region. This fast evolution leads to a radial size distribution showing a regular increase of grain size with decreasing distance from the star. At later times (Figs. 3c and d), we see a change in the profile shape due to differences in migration efficiency in different parts of the disk. The overall distribution then

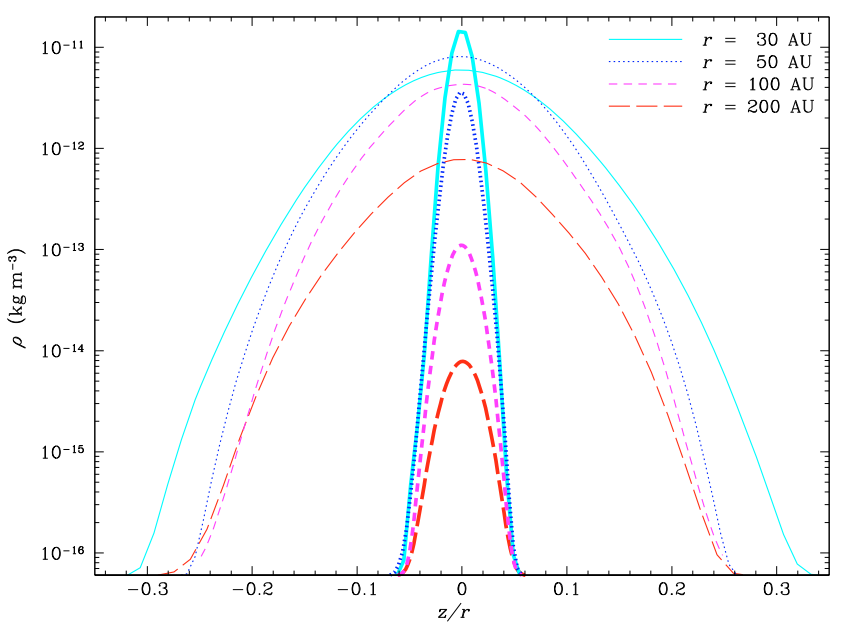

Fig. 2. Vertical (azimuthally averaged) profiles of the gas (thin lines) and dust (thick lines) densities at selected radii in the disk.

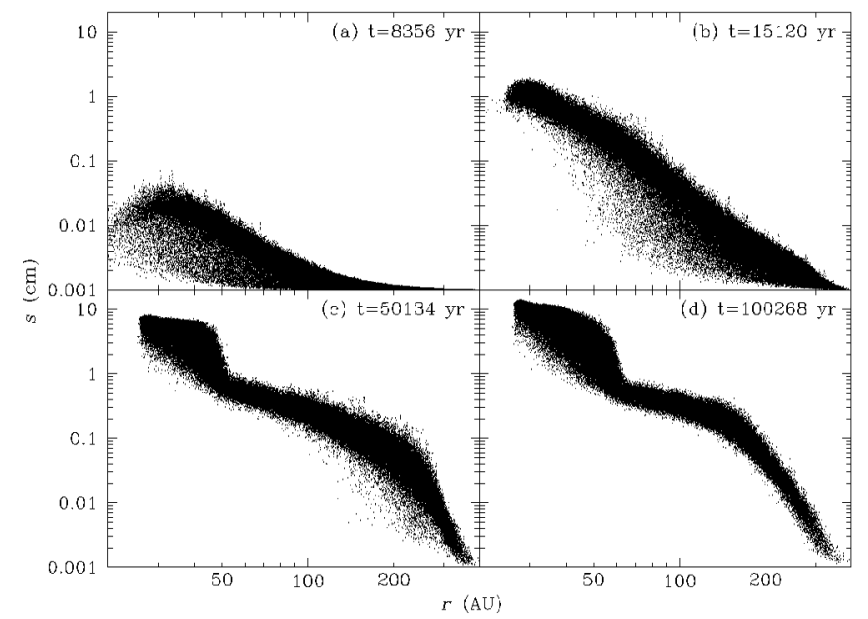

Fig. 3. Evolution of the radial grain size distribution for $s_{0}=10 \mu \mathrm{m}$.

evolves more slowly and shifts to larger sizes while keeping a roughly constant average slope.

In Fig. 4, we show the trajectories of seven individual particles in the $r-z$ plane for the $s_{0}=10 \mu \mathrm{m}$ case. The top panel shows the particles settling to the mid-plane followed by their radial migration and the bottom panel shows the grain growth during this process. We see three stages: particles grow as they settle to the mid-plane and then start their radial migration (this behaviour, seen for all particles in Fig. 4, was also reported by Haghighipour 2005), they then rapidly migrate inwards while growing very little (seen for P2, P3, P4, P5), and finally grow again while migrating very little (see for P1, P2, P3). The second stage of fast radial migration corresponds to the low slope seen in the central parts of the disk in Figs. $3 \mathrm{c}$ and d: grains spend less time there and accumulate in the inner disk, where they grow more efficiently due to the higher density.

The final size distribution (Fig. 3d) shows a population of large grains over $10 \mathrm{~cm}$ in the inner disk, whereas the grains stay well below $\mathrm{mm}$ sizes in the outer disk. The histogram of final grain sizes for the $s_{0}=10 \mu \mathrm{m}$ model (Fig. 5d) shows a bimodal distribution. The first peak corresponds to the end of the first growth stage identified in Fig. 4 where grains reach a size of about $3 \mathrm{~mm}$. The minimum around $8 \mathrm{~mm}$ is explained by the rapid migration of the second stage for grains of that size, which transports them to the very efficient growth region of the inner 


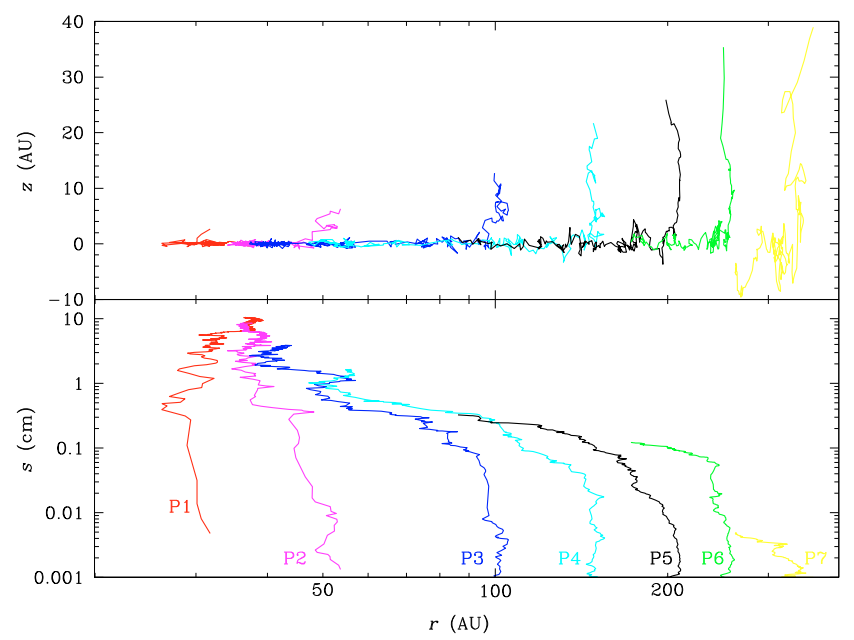

Fig. 4. Trajectories of individual particles for $s_{0}=10 \mu \mathrm{m}$.

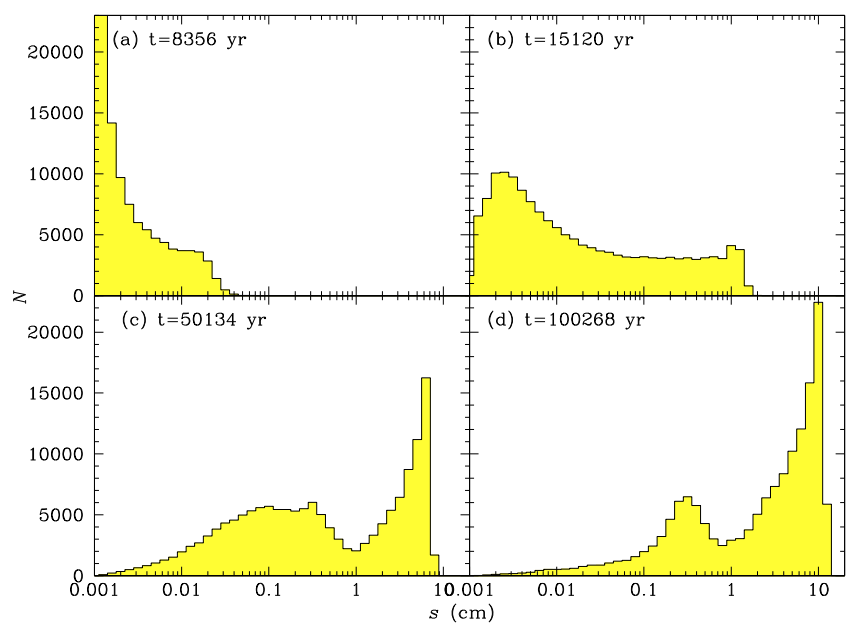

Fig. 5. Evolution of the histogram of grain sizes for $s_{0}=10 \mu \mathrm{m}$.

disk where they will populate the second peak around $10 \mathrm{~cm}$. As is to be expected from Eq. (1), the larger grains are found in the denser zones (see Fig. 1).

The histograms of grain sizes at different times displayed in Fig. 5 show that the signature of the rapid migration stage as a minimum around $8 \mathrm{~mm}$ appears only a few thousand years after the dust injection (Fig. 5b), as soon as the larger grains reach that size. It is visible thoughout the disk evolution, with a remarkably stable position (Figs. $5 \mathrm{c}$ and d).

Changing $s_{0}$ from $1 \mu \mathrm{m}$ to $1 \mathrm{~mm}$ only has an effect on the size distribution in the outer disk, where grains do not grow beyond a few $\mathrm{mm}$. In the rest of the disk, whatever their initial size, particles quickly reach the second stage and their subsequent evolution is similar to the $s_{0}=10 \mu \mathrm{m}$ case, leading to the same grain size distribution.

In this paper, we have restricted our study to disks with a large inner radius of $r_{\text {in }}=20 \mathrm{AU}$. For lower values, simulations are much slower due to the smaller drag timestep in the central higher density regions. Simulations with $r_{\text {in }}=3 \mathrm{AU}$ were run for $t=20000 \mathrm{yr}$ and show the same size distribution outside of $20 \mathrm{AU}$, with $s$ continuing to increase as $r$ decreases to $3 \mathrm{AU}$.

\section{Discussion}

Our results are in good agreement with those of SV97. Indeed, with a disk of comparable mass to ours and an $\alpha$ viscosity parameter of $10^{-2}$, they found that grain growth is more efficient in the inner disk, where grains reach sizes of $1 \mathrm{~cm}$ at $20 \mathrm{AU}$ after $3 \times 10^{5}$ years. They explain their results by the combined action of both growth and migration: grains grow, reach sizes where they decouple from the gas and then migrate, thereby altering the dust surface density and grain size. Equilibrium occurs when the global flux of migrating particles at a given radius vanishes. However, they do not resolve the change of slope in the grain size distribution between 50 and 150 AU. Further, their interpretation remains qualitative and does not recover the different growth stages we observe.

In order to interpret our results in a more quantitative way, we compare our simulations to an analytic expression of the resulting grain sizes derived under simplifying hypotheses. We first assume that $\bar{v} \ll V_{\mathrm{t}}$, i.e. that Sc is dominated by the effect of gas-dust coupling, determined by the value of the nondimensional stopping time $T_{\mathrm{s}}=\Omega_{\mathrm{K}} t_{\mathrm{s}}$. We introduce the nondimensional grain size $S=s / s_{\text {opt }}$, where

$s_{\mathrm{opt}}=\frac{C_{\mathrm{s}} \rho_{\mathrm{g}}}{\Omega_{\mathrm{K}} \rho_{\mathrm{d}}}$

is the optimal grain size for radial migration (for details see Fouchet et al. 2007), in order to write $\mathrm{Sc} \simeq 1+S$. Interestingly, $S=T_{\mathrm{s}}$ and the three drag regimes identified by BF05 are defined by $S \ll 1$ (dust strongly coupled to the gas), $S \sim 1$ (most efficient dust settling and migration) and $S \gg 1$ (dust decoupled from the gas).

To obtain an analytic solution for Eq. (1), we then neglect the temporal variation of all quantities except $s$. Since SV97 used constant values for Ro and $\alpha$, the only remaining variables are $\Omega_{\mathrm{K}}, \rho_{\mathrm{g}}, \hat{\rho}_{\mathrm{d}}$ and $C_{\mathrm{s}}$, which vary both in space and time. Assuming they are constant when following the evolution of the size of one particle amounts to assuming that, while it grows, the particle stays in the same position in the disk (therefore does not settle nor migrate) and that the disk structure does not evolve with time. While crude, this approximation is nonetheless useful in understanding the growth process and disentangling its effects from the complex interaction of those of the drag force. We define the dimensionless time

$T=\frac{t}{\tau}+2 \sqrt{S_{0}}\left(1+\frac{S_{0}}{3}\right)$, with $\tau=\frac{1}{\sqrt{2^{3 / 2} \operatorname{Ro} \alpha} \Omega_{\mathrm{K}}} \frac{\rho_{\mathrm{g}}}{\hat{\rho}_{\mathrm{d}}}$,

to rewrite Eq. (1) as

$\frac{\mathrm{d} S}{\mathrm{~d} T}=\frac{\sqrt{S}}{1+S}$

Its solution is

$S=\frac{\left(8+9 T^{2}+3 T \sqrt{16+9 T^{2}}\right)^{1 / 3}}{2}+\frac{2}{\left(8+9 T^{2}+3 T \sqrt{16+9 T^{2}}\right)^{1 / 3}}-2$

Three regimes can be isolated:

$\mid \begin{aligned} & S \ll 1 \Leftrightarrow T \ll 1: S \sim \frac{1}{4} T^{2} \\ & S \sim 1 \Leftrightarrow T \sim \frac{8}{3}: S=\frac{T}{2}-\frac{1}{3} \\ & S \gg 1 \Leftrightarrow T \gg 1: S \sim\left(\frac{3}{2} T\right)^{2 / 3},\end{aligned}$ 


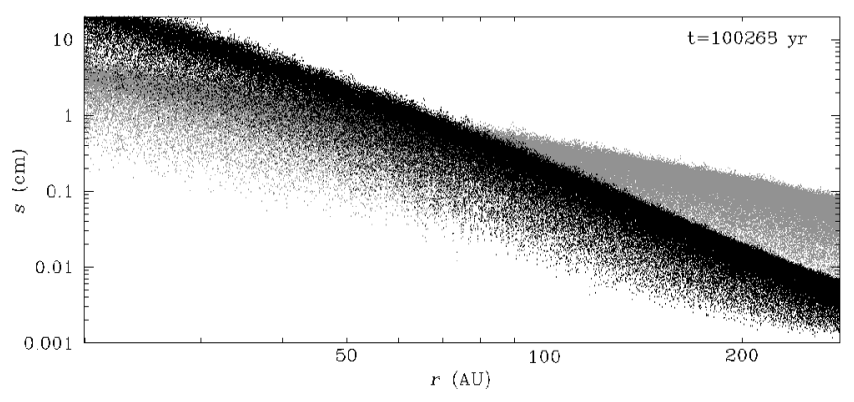

Fig. 6. Radial grain size distribution computed from Eq. (7) at the end time of our simulations, for $s_{0}=10 \mu \mathrm{m}$ (black) and optimal size for radial migration $s_{\text {opt }}$ (grey).

showing a fast growth for small sizes and a slower growth for larger sizes, consistent with what was seen in Fig. 3.

Although Eq. (7) associates one value of $S$ to a given value of $T$, the set of normalization quantities $\left(s_{\mathrm{opt}}, \tau\right)$ defining the non dimensional $S$ and $T$ is unique for each particle, and is computed with the disk parameters corresponding to its initial location. The resulting values of $s$ will therefore be different for all particles.

Starting from the same initial positions of dust particles of size $s_{0}$, this simple model allows us, instead of evolving their size with the code, to directly compute with Eq. (7) the resulting size (i.e. the size computed for the end time of our simulations) of each particle in the approximation of a fixed position during growth. The resulting grain size distribution is shown in Fig. 6 for $s_{0}=10 \mu \mathrm{m}$. As $s$ is proportional to $\rho_{\mathrm{g}}$ via $s_{\mathrm{opt}}$, the observed spread in resulting grain size at a given $r$ is caused by the spread in gas density for particles at different heights above the midplane. The resulting distribution reproduces the same grain size range as obtained at the end of the simulation, with decimetric grains in the inner disk, and has a slope close to that of the distribution in Fig. 3b. Of course, in that approximation, Eq. (7) cannot reproduce the effect of migration and therefore does not show the changes in slope that it causes, which were seen in Figs. $3 \mathrm{c}$ and $\mathrm{d}$.

The understanding of the growth process in the framework of our approximation which leads to Eq. (6) now allows us to reinterpret the results of grain growth in the presence of vertical settling and radial migration. For the range of $s_{0}$ values used in our simulations, $s_{0}<s_{\text {opt }}$ over most of the disk (see Fig. 6), and the grains are initially in the first regime of fast growth $(S \ll 1)$ identified in Sect. 4.2, and found in Eq. (8), corresponding to the strongly coupled drag regime, with very little migration. Outside of $\sim 150 \mathrm{AU}$, resulting grain sizes stay below $s_{\text {opt }}$ in the simulated timescale and grains do not reach the rapid migration stage. Indeed, the growth timescale $\tau \propto \Omega_{\mathrm{K}}^{-1} \propto r^{3 / 2}$ is longer in the outer disk. Inside of this radius, sizes do reach $s_{\text {opt }}$ (i.e. $S \sim 1$, corresponding to the intermediate drag regime) and grains go through a phase of fast inwards migration, explaining the low slope in the central region of Fig. $3 \mathrm{~d}$ and the stable location of the minimum in the histograms of grain sizes (Figs. $5 \mathrm{~b}-\mathrm{d}$ ). Indeed, $s_{\mathrm{opt}}$ depends on variables describing the gas disk, which does not evolve in the timescale of our simulations. Once they have grown to sizes greater than $s_{\text {opt }}$, the migration efficiency drops as dust starts to decouple from the gas (in the weak drag regime, defined by $S \gg 1$ ) and grains pile up inside of $\sim 50 \mathrm{AU}$ and continue to grow there, more slowly.

This result differs from that of Weidenschilling (1977), who models the particular case of the Minimum Mass Solar Nebula. With his disk parameters, he found that the grains with the largest radial migration velocity were meter-sized and that their consequent survival time in the nebula was much shorter than the disk lifetime. This led to the persisting (see, e.g., Natta et al. 2007) idea that growing grains which reached meter sizes rapidly fall onto the star (or more likely are evaporated in the inner disk), thus resulting in a potential problem for the planet formation process. This so-called "meter-size barrier" could be overcome if grains could grow to larger sizes within only a few hundred years, which would be very difficult according to the current understanding of solid particle aggregation (Blum 2006). Our simulations show that, in our less dense nebula representative of many observed T Tauri disks, growing grains can survive the fast migration stage (occurring for $\sim 8 \mathrm{~mm}$ grains in our disk conditions) and reach larger sizes with longer survival times, allowing for the possibility of planet formation inside of $\sim 50 \mathrm{AU}$.

\section{Conclusion}

We have implemented a mechanism able to treat grain growth in protoplanetary disks via the analytical expression of SV97 into our two-phase SPH code. We simulated for the first time the full $3 \mathrm{D}$ evolution of a typical T Tauri disk, following the simultaneous radial migration, vertical settling, and growth of solid particles. Their interplay is complex: dynamics affects grain growth by modifying local physical quantities such as density or relative velocity. Conversely, grain growth also acts on dust dynamics: where non-growing grains would either stay well mixed with the gas or settle and migrate according to their sizes (BF05), growing grains will go through various stages and produce totally different spatial distributions. They initially grow rapidly as they settle to the mid-plane, then experience a fast radial migration with little growth through the bulk of the disk, and finally pile-up in the inner disk where they grow more efficiently. This results in a bimodal distribution of grain sizes, with the largest grains found in the denser inner disk, where growth is most efficient. The survival times of the solid particles are longer than previously found, which has an implication on planet formation.

We find that grains grow very quickly: they reach decimetric size in $10^{5} \mathrm{yr}$. This is in general agreement with the results of Dullemond \& Dominik (2005) where shattering is neglected. They used the different approach of solving the Smoluchowski equation to study the growth of settling, but non-migrating, dust and in $10^{5} \mathrm{yr}$ formed grains of maximum sizes ranging from $1 \mathrm{~cm}$ to over $1 \mathrm{~m}$ depending on their model parameters. Similar to them, we also find that the small grains only survive in the very outer disk and are depleted too rapidly elsewhere to be consistent with infrared observations of disks, highlighting the importance of shattering.

In order to compute synthetic images from our simulations and compare them to the observations, one would have to assume that the collisional cascade resulting from the inclusion of shattering would produce a whole particle distribution from the maximum size at a given radius shown in Fig. 3d down to sub$\mu \mathrm{m}$ size, described by a quasi-steady power law as argued by Garaud (2007).

The method we used to treat grain growth can easily be applied to other analytical prescriptions. The development of a more detailed model is necessary for a realistic description of grain growth in protoplanetary disks. In addition to shattering, one needs to take into account other processes such as microscopic interactions between the grains, kinetic energy dissipation and grain porosity. This is the subject of a forthcoming paper.

Acknowledgements. We thank Yann Alibert for suggesting the use of the Stepinski \& Valageas (1997) approach. This research was partially supported 
by the Programme National de Physique Stellaire of CNRS/INSU, France, the Programme International de Coopération Scientifique (PICS) France-Australia in Astrophysics (Formation and Evolution of Structures), and the Swinburne University Research Development Grant Scheme. Simulations presented in this work were run on the Swinburne Supercomputer ${ }^{1}$. Images in Fig. 1 were made with SPLASH (Price 2007).

\section{References}

Apai, D., Pascucci, I., Sterzik, M. F., et al. 2004, A\&A, 426, L53

Barrière-Fouchet, L., Gonzalez, J.-F., Murray, J. R., Humble, R. J., \& Maddison, S. T. 2005, A\&A, 443, 185

Blum, J. 2006, Adv. Phys., 55, 881

Carpenter, J. M., Wolf, S., Schreyer, K., Launhardt, R., \& Henning, T. 2005, AJ, 129,1049

Chokshi, A., Tielens, A. G. G. M., \& Hollenbach, D. 1993, ApJ, 407, 806

Ciesla, F. J. 2007, ApJ, 654, L159

Dominik, C., Blum, J., Cuzzi, J. N., \& Wurm, G. 2007, in Protostars and Planets V, ed. B. Reipurth, D. Jewitt, \& K. Keil, 783

Dubrulle, B., Morfill, G., \& Sterzik, M. 1995, Icarus, 114, 237

Dullemond, C. P., \& Dominik, C. 2004, A\&A, 421, 1075

Dullemond, C. P., \& Dominik, C. 2005, A\&A, 434, 971

Fouchet, L., Maddison, S. T., Gonzalez, J.-F., \& Murray, J. R. 2007, A\&A, 474, 1037

Garaud, P. 2007, ApJ, 671, 2091

Garaud, P., Barrière-Fouchet, L., \& Lin, D. N. C. 2004, ApJ, 603, 292
Graham, J. R., Kalas, P. G., \& Matthews, B. C. 2007, ApJ, 654, 595 Haghighipour, N. 2005, MNRAS, 362, 1015

Haisch, Jr., K. E., Lada, E. A., \& Lada, C. J. 2001, ApJ, 553, L153

Hartmann, L., Calvet, N., Gullbring, E., \& D’Alessio, P. 1998, ApJ, 495, 385

Kessler-Silacci, J., Augereau, J.-C., Dullemond, C. P., et al. 2006, ApJ, 639, 275

King, A. R., Pringle, J. E., \& Livio, M. 2007, MNRAS, 376, 1740

Lommen, D., Wright, C. M., Maddison, S. T., et al. 2007, A\&A, 462, 211

Maddison, S. T., Fouchet, L., \& Gonzalez, J.-F. 2007, Ap\&SS, 311, 3

Muzerolle, J., Adame, L., D’Alessio, P., et al. 2006, ApJ, 643, 1003

Natta, A., Testi, L., Calvet, N., et al. 2007, in Protostars and Planets V, ed. B. Reipurth, D. Jewitt, \& K. Keil, 767

Nomura, H., \& Nakagawa, Y. 2006, ApJ, 640, 1099

Pinte, C., Fouchet, L., Ménard, F., Gonzalez, J.-F., \& Duchêne, G. 2007, A\&A, 469, 963

Price, D. 2007, PASA, 24, 159

Rodmann, J., Henning, T., Chandler, C. J., Mundy, L. G., \& Wilner, D. J. 2006, A\&A, 446, 211

Schräpler, R., \& Henning, T. 2004, ApJ, 614, 960

Shakura, N. I., \& Sunyaev, R. A. 1973, A\&A, 24, 337

Stepinski, T. F., \& Valageas, P. 1997, A\&A, 319, 1007

Suttner, G., \& Yorke, H. W. 2001, ApJ, 551, 461

Tanaka, H., Himeno, Y., \& Ida, S. 2005, ApJ, 625, 414

Weidenschilling, S. J. 1977, MNRAS, 180, 57

Weidenschilling, S. J. 1980, Icarus, 44, 172

Weidenschilling, S. J. 1997, Icarus, 127, 290

Youdin, A. N., \& Lithwick, Y. 2007, Icarus, 192, 588

${ }^{1}$ http://astronomy.swin. edu.au/supercomputing/ 